Editorial

\title{
Computational Methods and Applications to Simulate Water-Related Natural Hazards
}

\author{
Raffaele Albano $\left(\mathbb{D},{ }^{1}\right.$ Sauro Manenti $\left(\mathbb{D},{ }^{2}\right.$ José M. Domìnguez, ${ }^{3}$ Shaowu Li $\mathbb{D},{ }^{4}$ \\ and Dong Wang ${ }^{5,6}$ \\ ${ }^{1}$ School of Engineering, University of Basilicata, 85100 Potenza, Italy \\ ${ }^{2}$ Department of Civil Engineering and Architecture, University of Pavia, via Ferrata 3, 27100 Pavia, Italy \\ ${ }^{3}$ EPHYSLAB Environmental Physics Laboratory, Universidade de Vigo, As Lagoas 32004, Ourense, Spain \\ ${ }^{4}$ State Key Laboratory of Hydraulic Engineering Simulation and Safety, Tianjin University, Tianjin 300072, China \\ ${ }^{5}$ Department of Civil \& Environmental Engineering, National University of Singapore, 21 Lower Kent Ridge Road, \\ Singapore 119077 \\ ${ }^{6}$ College of Environmental Sciences and Engineering, Dalian Maritime University, Dalian 116000, China
}

Correspondence should be addressed to Raffaele Albano; raffaele.albano@unibas.it

Received 30 January 2020; Accepted 30 January 2020; Published 19 February 2020

Copyright (c) 2020 Raffaele Albano et al. This is an open access article distributed under the Creative Commons Attribution License, which permits unrestricted use, distribution, and reproduction in any medium, provided the original work is properly cited.

Thanks to the availability of high-performance computers, in the last few years, Computational Fluid Dynamics (CFD) has been widely applied to simulate natural hazards in the field of environmental and civil engineering [1]. In order to explore different risk scenarios and potential risk-reduction options and to address hazard related uncertainty, the development of numerical models represents an important task in supporting emergency response agencies, water resource managers, insurance companies, and other decision-makers [2]. The application purpose of CFD modeling requires contextual attention to the output variables of predictive interest and their time and space scales, the level of accuracy required considering eventually real-time data assimilation, and computational efficiency demands $[3,4]$.

The main aim of this special issue is to highlight the most recent advances in CFD modeling and its application related to water-related natural hazards and discusses future directions. Special focus is devoted to the modeling, handling of uncertainty, and the applications related to relevant natural hazard problems of practical and theoretical interest. A brief summary of all accepted papers is provided below.

In the paper by Y. Shi et al., they have investigated, through the numerical CFD test, the influence of bed surface roughness on the flow structure in an open channel. In particular, they have developed a CFD meshless method, such as a weakly compressible smoothed particle hydrodynamics (WCSPH) [5], for open-channel turbulence simulation. The proposed rough bed model is based on the ghost boundary particles (GBPs) [6]; it uses wall function for a small-scale rough boundary. The results outperform the traditional mixed-length-based subparticle scale (SPS) turbulence model [7] by obtaining small numerical errors in the mainstream and near-surface region but it is found that errors varied widely for the inner region; the authors showed that the latter error is positively correlated with the channel bed slope and the equivalent roughness. On the basis of these results, the study proposes empirical formulae to calculate the ghost particle velocity under different hydraulic conditions using data mining.

C. Zhen et al. have investigated the fatigue failure for trimaran ship, which is actually considered as a good alternative to monohulls in high-speed transportation and in naval applications [8]. In particular, they have presented a simplified fatigue analysis of trimaran cross-deck structure based on three-dimensional (3D) linear potential flow theory and global finite element (FE) analysis for wave loads and stress transfer functions calculation; at the same time, a stochastic spectral fatigue analysis is carried out considering 
the weighted wave heading factors. Then, the authors have demonstrated the application of the proposed method by simulating the fatigue lives of a few hot spots. The outcome of the study is to extend Lloyd's Register Rules guideline [9] for the structural design of these types of ships adding more specifications on the fatigue characteristic of trimaran crossdeck structure, which includes the most dangerous fatigue position and the fatigue damage proportion of various wave heading directions.

In the paper by J. Han et al., they have investigated, through the finite element numerical simulations, the mechanism of fracturing in shaft lining, i.e., the passageway of mine production, due to high-pressure pore water. In particular, the authors have adopted the ANSYS numerical simulation (https://www.ansys.com) to obtain the volume change of the cubic model under the pore water pressure. Based on the calculation results, they have derived an approximate analytical solution of the hydraulic expansion coefficient by means of the multivariate nonlinear regression method. Analyzing the interaction between the pore water pressure and the porous rock and shaft lining, the authors found that the hydraulic expansion effect on the surrounding rock increases with its porosity and decreases with its elastic modulus and Poisson's ratio.

S. Gao et al. have used ANSYS finite element numerical software to simulate thermodynamic performance in the fireproof sealing, which is widely employed to limit the scale of fire in multistory building, commercial building, industrial building, medical building, and other types of public buildings. They have estimated the main thermal parameters, including temperature field, thermal flux, and thermal gradient, for two fireproof sealing models that differ in terms of model shape and the initial fire surface. The authors, through the finite element numerical simulations, have evaluated the performances of the two models in terms of fire protection concluding that the rectangle model outperforms the " $\mathrm{T}$ " shape model. The conclusions of the study are meaningful to improve the thermodynamic performance of the fire-proof sealing in the converter station.

J. F. Liang and X. Wang have proposed a consistent Riccati expansion method [10] for obtaining interaction solutions, such as soliton-soliton, multiple resonant soliton, soliton-cosine wave, and soliton-cnoidal wave solutions, to the modified Korteweg-de Vries (mKdV) equation [11] that could be used in the water wave system. In particular, they have demonstrated that interaction solutions such as the soliton-tangent wave solution cannot be constructed for the $\mathrm{mKdV}$ equation. Moreover, they have shown that soliton and soliton-soliton wave interaction solutions can be derived from the soliton-cnoidal wave interaction solution by making the limit of the modulus approach either 0 or 1 (i.e., lower or upper bounds for the modulus in the Jacobi elliptical function).

In the paper by N. J. Lim and S. A. Brandt, they have analyzed how the change of resolution in the adopted DEM (Digital Elevation Model) in combination with the roughness parameter can affect the performances of hydraulic numerical modelling for areas with different topographies.
In particular, they have estimated the uncertainties in terms of flood extent, respectively, for one-dimensional (i.e., HEC-RAS [12]) and a two-dimensional models (i.e., CAESAR-LISFLOOD [13]) in flat and mountain river basins by comparing the simulations result with the observed flood data of historical events. The results showed that poor-resolution DEMs might produce a global high feature agreement score with historical data but may fail to provide good flood extent estimations locally, particularly in flat areas. Instead, high-resolution DEMs (1 to $5 \mathrm{~m}$ ) remain advantageous for modelling as they represent better the topography of the study area but it is important to carefully calibrate the models by the use of the roughness parameter. The outcomes of the study confirm that the knowledge of the magnitude and source of uncertainties helps to improve assessments and leads to better inform decisions on flood risk mitigation alternatives [14]. Moreover, numerical models need to be controlled, for example, through a calibration process that adjusts model parameters, assumptions, or equations to optimize concordance between observed data and model predictions [15].

In this context, remote sensing is a low-cost technology that represents an important source of information in the water-related hazards and in water resource management fields [16]. K. Ennouri and A. Kallel have reviewed the advantages and limitations (e.g., spatiotemporal constraints) of the main remote sensing sensors, indices, and algorithms for image analysis (e.g., postprocessing techniques, classification algorithms, and evaluation techniques) in the field of crop condition assessment. They have discussed and highlighted how the remote sensing monitoring plays a central role in diagnosing climate and management impacts on agricultural systems.

H. Liu et al. focused their study on soil erosion risk assessment and soil conservation planning. In particular, the authors have analyzed the temporal trends and spatial patterns of rainfall erosivity ( $R$ factor) for a large basin in China (i.e., Three Gorges Reservoir Area) during the period of 1960-2010. They have used the Mann-Kendall test [17] and co-kriging interpolation to analyzed data of meteorological stations and hydrological rain and gauging stations aiming to examine the spatial distribution of annual and seasonal $R$ factor and to characterize the temporal trends of $R$ factor for different time scales in the light of the same data span. The outcomes highlighted in this study can support watershed planning of soil conservation measures in the Three Gorges Reservoir Area.

\section{Conflicts of Interest}

The editors declare that they have no conflicts of interest regarding the publication of this Special Issue.

\section{Acknowledgments}

The guest editors would like to thank all the authors for their contributions and the reviewers for their timely and constructive feedback. We hope this special issue can 
contribute to advancements of the scientific community in the field of engineering and natural hazard assessment and management.
[15] S. Scaprino, R. Albano, A. Cantisani, L. Mancusi, A. Sole, and G. Milillo, "Multitemporal SAR data and 2D hydrodynamic model flood scenario dynamics assessment," ISPRS International Journal of Geo-Information, vol. 7, no. 105, 2018.

[16] R. Albano and A. Sole, "Geospatial methods and tools for natural risk management and communications," ISPRS International Journal of Geo-Information, vol. 7, no. 12, p. 470, 2018.

[17] H. B. Mann, "Nonparametric tests against trend," Econometrica, vol. 13, no. 3, pp. 245-259, 1945.

\section{References}

[1] S. Manenti, D. Wang, J. M. Domínguez, S. Li, A. Amicarelli, and R. Albano, "SPH modeling of water-related natural hazards," Water, vol. 11, no. 9, p. 1875, 2019.

[2] R. Albano, L. Mancusi, A. Sole, and J. Adamowski, "FloodRisk: a collaborative free and open-source software for flood risk analysis," Geomatics Natural Hazard and Risk, vol. 8, no. 2, pp. 1812-1832, 2017.

[3] R. Albano, D. Mirauda, A. Sole, and J. Adamowski, "Modeling large floating bodies in urban floods via a smoothed particle hydrodynamics model," Journal of Hydrology, vol. 541, pp. 344-358, 2016.

[4] S. Manenti, A. Amicarelli, and S. Todeschini, "WCSPH with limiting viscosity for modeling landslide hazard at the slopes of artificial reservoir," Water, vol. 10, no. 4, p. 515, 2018.

[5] A. Amicarelli, R. Albano, D. Mirauda, G. Agate, A. Sole, and R. Guandalini, "A smoothed particle hydrodynamics model for 3D solid body transport in free surface flows," Computers \& Fluids, vol. 116, pp. 205-228, 2015.

[6] L. Fu and Y.-C. Jin, "A mesh-free method boundary condition technique in open channel flow simulation," Journal of $\mathrm{Hy}$ draulic Research, vol. 51, no. 2, pp. 174-185, 2013.

[7] H. Gotoh, T. Shibahara, and T. Sakai, "Sub-particle-scale turbulence model for the MPS method Lagrangian flow model for hydraulic engineering," Advanced Methods for Computational Fluid Dynamics, vol. 9, pp. 339-347, 2001.

[8] T. Armstrong, "On the performance of a large high-speed trimaran," Australian Journal of Mechanical Engineering, vol. 3, no. 2, pp. 123-131, 2006.

[9] L.'s Register, Rules for the Classification of Trimarans, Lloyd's Register, London, UK, 2006.

[10] S. Y. Lou, "Consistent Riccati expansion for integrable systems," Studies in Applied Mathematics, vol. 134, no. 3, pp. 372-402, 2015.

[11] R. Hirota, "Exact solution of the korteweg-de vries equation for multiple collisions of solitons," Physical Review Letters, vol. 27, no. 18, pp. 1192-1194, 1971.

[12] T. J. Coulthard, J. C. Neal, P. D. Bates, J. Ramirez, G. A. M. de Almeida, and G. R. Hanrock, "Integrating the LISFLOOD-FP 2D hydrodynamic model with the CAESAR model: implications for modelling landscape evolution," Earth Surface Processes and Landforms, vol. 38, no. 15, pp. 18971906, 2013.

[13] US Army Corps of Engineers Hydrologic Engineering Center, "HEC-RAS: river analysis system," User's Manual (V. 4.0), US Army Corps of Engineers Hydrologic Engineering Center, Davis, CA, USA, 2008.

[14] R. Albano, A. Sole, J. Adamowski, A. Perrone, and A. Inam, "Using floodrisk GIS freeware for uncertainty analysis of direct economic flood damages in Italy," International Journal of Applied Earth Observation and Geoinformation, vol. 73, pp. 220-229, 2018. 